\title{
Microrecording and image-guided stereotactic biopsy of deep-seated brain tumors
}

\author{
Keiya lijima, MD, ${ }^{1}$ Masafumi Hirato, MD, PhD, ${ }^{1}$ Takaaki Miyagishima, MD, PhD, ${ }^{1}$ \\ Keishi Horiguchi, MD, PhD, ${ }^{1}$ Kenichi Sugawara, MD, PhD, ${ }^{1}$ Junko Hirato, MD, PhD, ${ }^{3}$ \\ Hideaki Yokoo, MD, PhD, ${ }^{2}$ and Yuhei Yoshimoto, MD, PhD' \\ Departments of ${ }^{1}$ Neurosurgery and ${ }^{2}$ Human Pathology, Gunma University Graduate School of Medicine; and \\ ${ }^{3}$ Clinical Department of Pathology, Gunma University Hospital, Maebashi, Gunma, Japan
}

\begin{abstract}
OBJECT Image-guided stereotactic brain tumor biopsy cannot easily obtain samples of small deep-seated tumor or selectively sample the most viable region of malignant tumor. Image-guided stereotactic biopsy in combination with depth microrecording was evaluated to solve such problems.

METHODS Operative records, MRI findings, and pathological specimens were evaluated in 12 patients with small deep-seated brain tumor, in which image-guided stereotactic biopsy was performed with the aid of depth microrecording. The tumors were located in the caudate nucleus (1 patient), thalamus (7 patients), midbrain (2 patients), and cortex (2 patients). Surgery was performed with a frameless stereotactic system in 3 patients and with a frame-based stereotactic system in 9 patients. Microrecording was performed to study the electrical activities along the trajectory in the deep brain structures and the tumor. The correlations were studied between the electrophysiological, MRI, and pathological findings. Thirty-two patients with surface or large brain tumor were also studied, in whom image-guided stereotactic biopsy without microrecording was performed.
\end{abstract}

RESULTS The diagnostic yield in the group with microrecording was 100\% (low-grade glioma 4, high-grade glioma 4 , diffuse large B-cell lymphoma 3, and germinoma 1), which was comparable to $93.8 \%$ in the group without microrecording. The postoperative complication rate was as low as that of the conventional image-guided method without using microelectrode recording, and the mortality rate was $0 \%$, although the target lesions were small and deep-seated in all cases. Depth microrecording revealed disappearance of neural activity in the tumor regardless of the tumor type. Neural activity began to decrease from $6.3 \pm 4.5 \mathrm{~mm}$ (mean $\pm \mathrm{SD}$ ) above the point of complete disappearance along the trajectory. Burst discharges were observed in 6 of the 12 cases, from $3 \pm 1.4 \mathrm{~mm}$ above the point of decrease of neural activity. Injury discharges were often found at $0.5-1 \mathrm{~mm}$ along the trajectory between the area of decreased and disappeared neural activity. Close correlations between electrophysiological, MRI, and histological findings could be found in some cases.

CONCLUSIONS Image-guided stereotactic biopsy performed using depth microrecording was safe, it provided accurate positional information in real time, and it could distinguish the tumor from brain structures during surgery. Moreover, this technique has potential for studying the epileptogenicity of the brain tumor.

http://thejns.org/doi/abs/10.3171/2014.10.JNS14963

KEY WORDS stereotactic biopsy; image guided; depth microrecording; burst; tumor-brain interface; epileptogenicity; stereotactic radiosurgery

$\mathrm{S}$ TEREOTACTIC neurosurgery, first described by Zernov in $1889,{ }^{41}$ has been improved in accuracy by the successive development of the stereotactic frame, image diagnosis, image navigation system, and robotic surgery. $3,12,17,22,23,25,37,38$ Stereotactic biopsy has been gradually applied to removal of brain tumor in cases in which resection is difficult and histological diagnosis is needed. ${ }^{18,19,21}$
Recently, excellent surgical results have been achieved with image-guided stereotactic biopsy performed using intraoperative MRI or ultrasonography., 1,5,31 However, stereotactic biopsy of the brain tumor, particularly of deepseated small lesions, must differentiate the tumor from the surrounding brain tissue to obtain viable tissue samples. Moreover, any neurological deficit caused by injury to the

ABBREVIATIONS DLBCL = diffuse large B-cell lymphoma; HGG = high-grade glioma; LGG = low-grade glioma.

SUBMITTED April 30, 2014. ACCEPTED October 6, 2014.

INCLUDE WHEN CITING Published online March 27, 2015; DOI: 10.3171/2014.10.JNS14963.

DISCLOSURE The authors report no conflict of interest concerning the materials or methods used in this study or the findings specified in this paper. 
surrounding normal brain structures must be minimized. Conventional image-guided stereotactic biopsy cannot easily achieve selective removal of the most malignant region of heterogeneous tumor. In addition, specific cases present with difficulties, such as thalamic or brainstem lesion (particularly if the tumor is small), with associated higher morbidity and mortality. ${ }^{8,14}$ Because the conventional image-guided stereotactic biopsy depends only on the anatomical data, certain difficulties may sometimes arise.

Fifty years ago in France, the microrecording technique was systematically introduced into the stereotactic procedure in humans to study the electrical activity of thalamic neurons. ${ }^{15,24,26}$ This technique provided precise and direct information about functional neuroanatomy in the deep subcortical structures and is recognized as one of the most reliable techniques to determine the final target point and to correct the inaccuracy of stereotactic procedures in individual cases. Although interpretation of microrecording data in the deep brain structures is sometimes difficult for neurosurgeons, our preliminary study showed that neural activity disappeared in the tumor, ${ }^{29}$ which indicates that the tumor location could simply be identified based on the presence or absence of electrical activity. Therefore, the present method may enable neurosurgeons to perform this type of surgery even without detailed knowledge about the neuronal firing pattern of individual brain structures.

To resolve these problems, we have developed a method of image-guided stereotactic biopsy sampling that is performed with the aid of depth microrecording. The present study assessed the accuracy and safety of this method and investigated the correlations between the electrophysiological, MRI, and histological findings.

\section{Methods}

\section{Patient Selection}

Stereotactic biopsy was performed in 44 patients (19 female and 25 male patients) with various intracerebral lesions at our department between 1998 and 2013. Stereotactic biopsy was performed with the aid of depth microrecording for deep-seated and small lesions in 12 patients -5 females and 7 males - between the ages of 7 and 75 years (mean 47.9 years). Patient profile; tumor location, side, and diameter; operative method and time; and histological diagnosis are summarized in Table 1 . The tumors were located in the caudate nucleus (1 patient), thalamus (7 patients), midbrain (2 patients), and cortex (2 patients). Patients underwent T1-weighted MRI with addition of $\mathrm{Gd}$, which showed tumor diameters of $10-45 \mathrm{~mm}$ (mean $20.8 \mathrm{~mm}$ ) (Fig. 1 left). The tumor vasculature and biopsy trajectory were evaluated with MR angiography and 3D CT angiography before surgery. Cerebral digital subtraction angiography was performed as needed. Stereotactic biopsy for surface or large lesions without microrecording was performed in the other 32 patients -14 females and 18 males-between the ages of 9 and 82 years (mean 62.9 years). Tumor diameter, location, diagnostic yield, histological diagnosis, complications, and operating time are summarized in Fig. 1 right. We obtained informed con- sent for the present method of treatment from all patients before surgery.

\section{Surgical Procedure}

Surgery with the aid of microrecording was performed with a frameless stereotactic system (NEURO-SAT, Mitaka Kohki Co., Ltd.) in 3 patients, and with a frame-based stereotactic system (Leksell stereotactic frame type G, Elekta Instruments) in 9 patients (Table 1). Surgery without microrecording was performed with a frame-based stereotactic system in all 32 patients.

\section{Frameless Stereotactic System With Microrecording}

Preoperative T1-weighted MR imaging with addition of Gd was performed using a 1.5-T MRI scanner (SIGNA HTxt Ver23, General Electric Systems) with 2-mm gapless slices and 3 fiducial markers. The MRI data were input into the NEURO-SAT system. The target point was selected near the center of the tumor based on the findings of all imaging methods, including PET. The patient was placed supine in all cases. After introduction of general anesthesia with propofol, a standard bur hole was made. The electrode was inserted from the cortex into the tumor in Cases 1 and 2. A guide needle was inserted with a mandolin up to $31 \mathrm{~mm}$ from the target point in Case 3. The mandolin was then changed to a microelectrode and electrical activity was recorded from this point. The biopsy specimen was obtained using the biopsy needle at the point considered to be tumor tissue from depth microrecording. The biopsy needle consisted of a double cannula, with outer diameter of $2 \mathrm{~mm}$ and inner diameter of $1.5 \mathrm{~mm}$, with a $10-\mathrm{mm}$ side-hole length. After setting the center of the biopsy needle at the intended target point, the side hole was opened and sample tissue was aspirated into the cannula by applying negative pressure with an attached syringe. Then the side hole was closed to cut and obtain sample tissue. Two to 4 specimens were obtained at the same point by rotating the side hole. One or 2 samples were used for intraoperative pathological examination of rapid-frozen sections. The other samples were sent for pathological examination of permanent sections.

\section{Frame-Based Stereotactic System With Microrecording}

In this subset of 9 patients surgery was conducted under local anesthesia in 8 patients, and under general anesthesia in Case 9, who was a 7-year-old child and could not remain still. Surgery was performed with the patient supine in all cases. After setting of the Leksell stereotactic frame type G, MRI was performed as described above. A $4-\mathrm{cm}$ skin incision and 2-cm craniostomy were made at the point $9 \mathrm{~cm}$ superior to the nasion and $3 \mathrm{~cm}$ lateral from the midline. The coordinates of the tumor center were calculated after inputting the MRI data into the image navigation system (StealthStation Tria containing Framelink Software, Medtronic). A guide needle with a mandolin was inserted up to the point $15-35 \mathrm{~mm}$ above the target. The mandolin was removed at this point and a microelectrode was inserted using a micromanipulator to record the electrical activity in the brain. After the tumor location was estimated based on the electrophysiological recording 
TABLE 1. Patient and tumor characteristics, operative method and time, and histological diagnosis in 12 patients with deep-seated brain tumors

\begin{tabular}{|c|c|c|c|c|c|c|c|c|}
\hline $\begin{array}{l}\text { Case } \\
\text { No. }\end{array}$ & $\begin{array}{c}\text { Age (yrs), } \\
\text { Sex }\end{array}$ & Tumor Location & Side & $\begin{array}{l}\text { Diam of } \\
\text { EL (mm) }\end{array}$ & $\begin{array}{l}\text { Stereotactic } \\
\text { System }\end{array}$ & Anesthesia & $\begin{array}{c}\text { Operating } \\
\text { Time }\end{array}$ & Diagnosis \\
\hline 1 & $19, \mathrm{~F}$ & Thalamus & $\mathrm{Rt}$ & 31 & Frameless & General & $2 \mathrm{hrs}, 5 \mathrm{~min}$ & Anaplastic oligodendroglioma \\
\hline 2 & $62, F$ & Cortex & $\mathrm{Rt}$ & 30 & Frameless & General & $2 \mathrm{hrs}, 10 \mathrm{~min}$ & Oligodendroglioma \\
\hline 3 & $45, F$ & Cortex & Rt & 10 & Frameless & General & $1 \mathrm{hr}, 40 \mathrm{~min}$ & Anaplastic oligoastrocytoma \\
\hline 4 & $55, M$ & Thalamus & $\mathrm{Lt}$ & 12 & Frame based & Local & $3 \mathrm{hrs}, 39 \mathrm{~min}$ & DLBCL \\
\hline 5 & $65, M$ & Thalamus & $\mathrm{Rt}$ & 15 & Frame based & Local & $4 \mathrm{hrs}, 0 \mathrm{~min}$ & Anaplastic astrocytoma \\
\hline 6 & $24, M$ & Thalamus & $\mathrm{Lt}$ & 12 & Frame based & Local & $3 \mathrm{hrs}, 25 \mathrm{~min}$ & Germinoma \\
\hline 7 & $17, M$ & Thalamus & $\mathrm{Rt}$ & 15 & Frame based & Local & $2 \mathrm{hrs}, 22 \mathrm{~min}$ & Pilocytic astrocytoma \\
\hline 8 & $73, \mathrm{~F}$ & Caudate-thalamus & $\mathrm{Lt}$ & 24 & Frame based & Local & $2 \mathrm{hrs}, 33 \mathrm{~min}$ & Glioblastoma \\
\hline 9 & $7, \mathrm{M}$ & Thalamus & $\mathrm{Lt}$ & 10 & Frame based & General & $4 \mathrm{hrs}, 3 \mathrm{~min}$ & Ganglioglioma \\
\hline 10 & $66, M$ & Midbrain & Lt & 30 & Frame based & Local & $3 \mathrm{hrs}, 35 \mathrm{~min}$ & Pilocytic astrocytoma \\
\hline 11 & $67, \mathrm{~F}$ & Midbrain & $\mathrm{Lt}$ & 15 & Frame based & Local & $3 \mathrm{hrs}, 13 \mathrm{~min}$ & DLBCL \\
\hline 12 & $75, M$ & Thalamus & $\mathrm{Lt}$ & 45 & Frame based & Local & $2 \mathrm{hrs}, 35 \mathrm{~min}$ & DLBCL \\
\hline
\end{tabular}

Diam = diameter; $\mathrm{EL}=$ enhanced lesion

data, the microelectrode was removed and a biopsy needle (outer diameter $2 \mathrm{~mm}$ and inner diameter $1.5 \mathrm{~mm}$, sidehole length $10 \mathrm{~mm}$ ) was inserted. Tissue samples were obtained by suction and cutting as described above. One or 2 samples of tumor tissue were used for intraoperative pathological examination of rapid-frozen sections in 8 patients (Cases 4-9, 11, and 12). All other samples, including that obtained in Case 10, were sent for pathological examination of permanent sections.

\section{Microrecording Protocol}

Microrecording was performed to study the electrical activities of the deep brain structures and disappearance of electrical activity in the tumor ${ }^{29}$ in every case. These findings provided adequate information about the position of the electrode within the brain and the tumor to determine the final point for suction biopsy. We used a bipolar concentric needle-type electrode (steel-steel; outer diameter $0.4 \mathrm{~mm}$, tip approximately $10 \mu \mathrm{m}$, interpolar distance approximately $500 \mu \mathrm{m}$, with electrical resistance less than $100 \mathrm{k} \Omega$ ). Not only the unitary spike discharge but also the global background activity can be recorded using this electrode. The electrode was inserted from the cortex into the tumor in Cases 1 and 2. Except in these cases, the guide needle was advanced manually with a mandolin from the cortex to a point $15-35 \mathrm{~mm}$ from the target to avoid bleeding in the cortex. The microelectrode was then advanced from this point to the target by using a pulse motor-driven micromanipulator. The low-bandpass filter was used as needed. The usual trajectory from the prefrontal area to the thalamic tumor passed through the caudate nucleus, internal capsule, and thalamus. Our previous preliminary study revealed that these subcortical structures have characteristic electrical activity, whereas the tumor showed no detectable electrical activity, ${ }^{29}$ so that we could distinguish the tumor clearly from the surrounding normal structure. The changes in the electrical activity pattern were similar for tumor in the midbrain, so that we could distinguish such tumors in the same way. Acquisition of the microrecording data required approximately 20 minutes in all cases.

\section{Correlations Between Electrophysiological, MRI, and Pathological Findings}

We studied the correlations between the electrophysio-

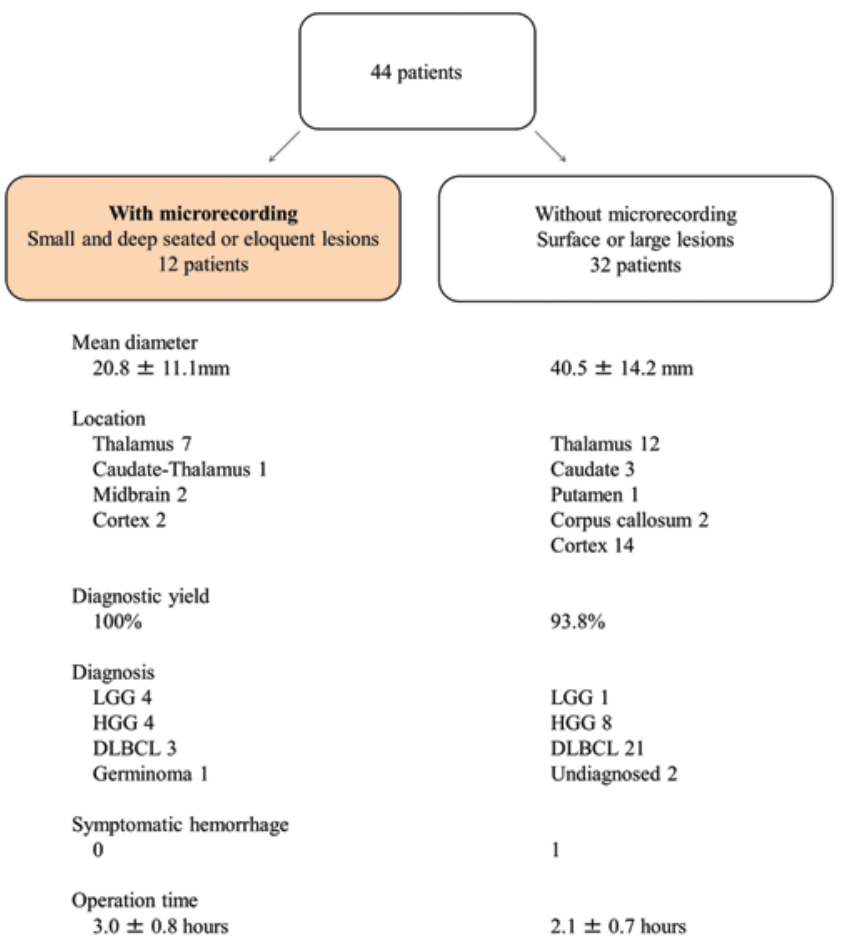

FIG. 1. Profiles of 44 patients who underwent image-guided stereotactic biopsy with (left) and without (right) the aid of depth microrecording. The mean diameter, location of tumor, diagnostic yield, histological diagnosis, number of symptomatic hemorrhages, and operating time in each group are shown from top to bottom below each box. Figure is available in color online only. 
logical, MRI, and pathological findings. The electrophysiological findings were classified into 4 categories: stable neural activity; burst (irregular) neural activity; decreased neural activity (sudden decrease of neural activity below one-half amplitude of stable); and disappeared neural activity. The MRI findings were classified into 4 categories: 1) normal appearance; 2) high intensity on $T 2$-weighted imaging without enhancement with Gd; 3) enhancement with Gd; and 4) low intensity on T1-weighted imaging. Additionally, the cases were classified into burst type (Fig. 2A) and simple type (Fig. 3A) depending on the presence or absence of burst neural activity. In several cases, surgical specimens were obtained from different regions in the same tumor in which different electrophysiological findings were observed.

\section{Management After Surgery}

Head CT scanning was performed just after the operation, and MRI with Gd was performed on the day after surgery to confirm the site of sampling of the tumor.

\section{Results}

\section{Histological Diagnosis and Diagnostic Yield}

Definitive histological diagnoses were obtained in all 12 patients who underwent surgery with microrecording, with a diagnostic yield per patient of $100 \%$. The neoplastic diagnosis included low-grade glioma (LGG) (4 patients), high-grade glioma (HGG) (4 patients), diffuse large B-cell lymphoma (DLBCL) (3 patients), and germinoma (1 patient) (Table 1, Fig. 1 left). Definitive histological diagnoses were obtained in 30 of the 32 patients who underwent surgery without microrecording, with a diagnostic yield per patient of $93.8 \%$. Neuropathological examination was not conclusive in 2 patients. The diagnosis was LGG in 1 patient, HGG in 8 patients, and DLBCL in 21 patients (Fig. 1 right).

\section{Postoperative Complications}

Transient sensory disturbance was observed in 1 patient with midbrain tumor (Case 11) just after surgery with microrecording. No other postoperative complications occurred. No permanent complication, symptomatic cerebral hemorrhage, or postoperative death was observed. Symptomatic hemorrhage and transient motor palsy occurred in 1 patient each, but no postoperative death occurred in patients treated surgically without microrecording.

\section{Electrophysiological Findings Along the Trajectory}

High and stable neural activity was observed in the caudate nucleus or thalamus in patients with thalamic or midbrain tumor. Low positive neural activity was observed in the internal capsule. Stable thalamic neural activity was observed along the trajectory in 9 patients (Cases 1, 4-7, and 9-12). Neural activity had disappeared within the tumor in all cases. Neural activity began to decrease from $6.3 \pm 4.5 \mathrm{~mm}$ (mean $\pm \mathrm{SD}$, range $2-18.4 \mathrm{~mm}$ ) above the
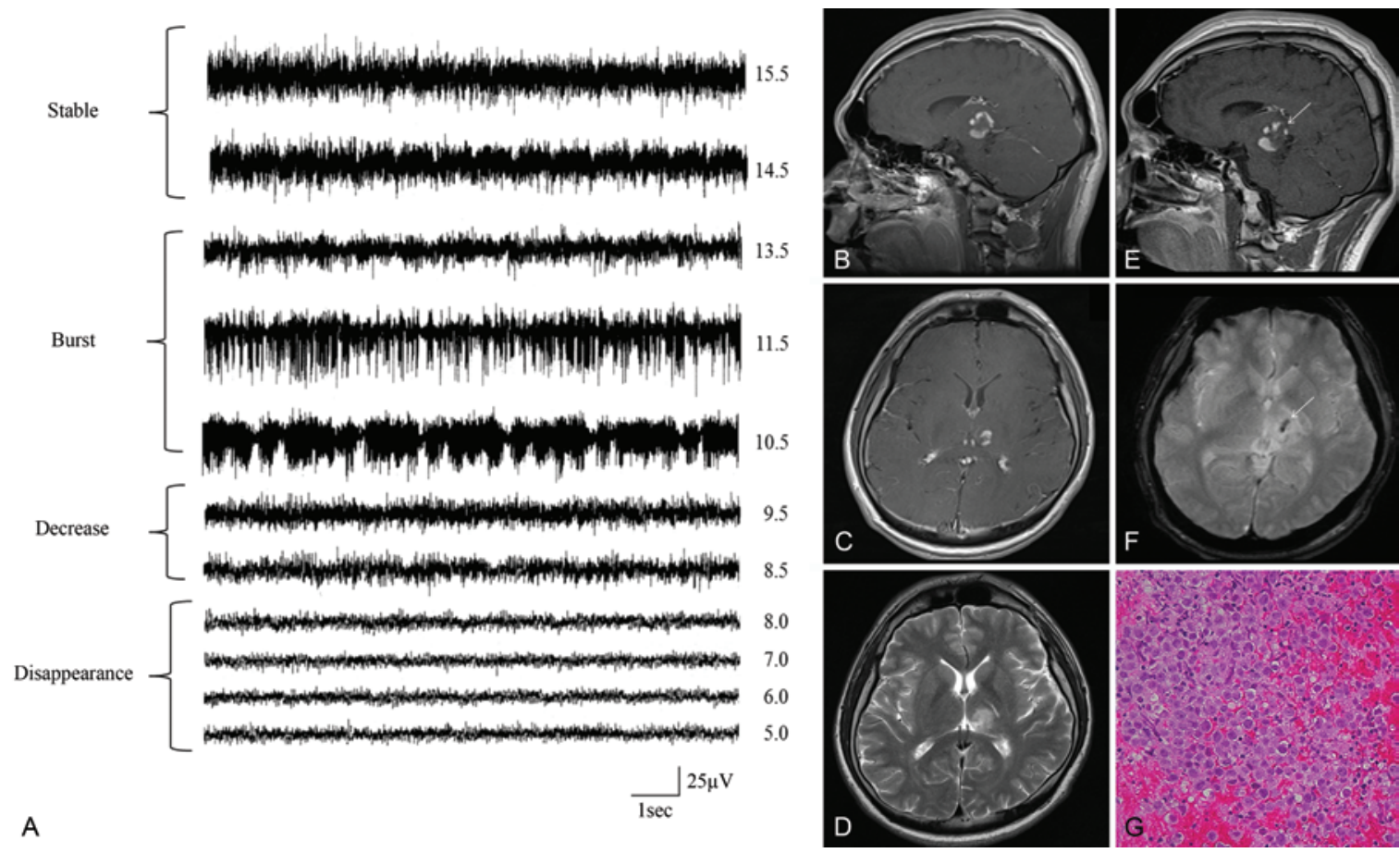

FIG. 2. Case 6. Electrophysiological, MRI, and pathological findings. A: Neural activity recording along the trajectory. Numerical value on the right side of the recording data shows distance from the target point in millimeters. B-D: Preoperative sagittal (B) and axial (C) T1-weighted MR images with Gd and axial T2-weighted MR image (D). E and F: Postoperative sagittal T1-weighted MR image with Gd (E) and axial T2*-weighted MR image (F). Arrows show sampling area of the tumor. G: Photomicrograph of sample obtained for histological examination demonstrating typical germinoma. $\mathrm{H} \& \mathrm{E}$, original magnification $\times 40$. Figure is available in color online only. 


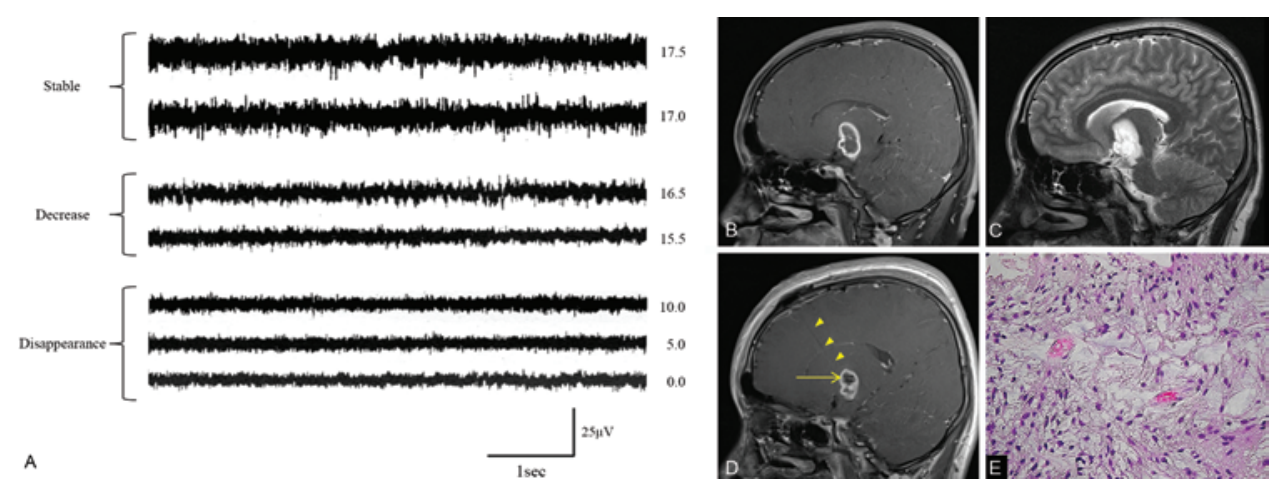

FIG. 3. Case 7. Electrophysiological, MRI, and pathological findings. A: Neural activity recording along the trajectory. B and C: Preoperative sagittal T1-weighted MR image with $\mathrm{Gd}(\mathrm{B})$ and T2-weighted MR image (C). D: Postoperative sagittal T1-weighted MR image with Gd. Arrow shows sampling area of the tumor and arrowheads show the trajectory of the biopsy needle. E: Photomicrograph of a sample obtained for histological examination demonstrating typical pilocytic astrocytoma. $\mathrm{H} \& \mathrm{E}$, original magnification $\times 40$. Figure is available in color online only.

point of complete disappearance of neural activity in all cases. Additionally, irregular burst discharges were observed from $3 \pm 1.4 \mathrm{~mm}$ (mean $\pm \mathrm{SD}$, range $1.5-4.5 \mathrm{~mm}$ ) above the point of decrease of neural activity in 6 burst type cases (Cases 1, 4-6, 11, and 12). Injury discharges, which were found only during insertion of the electrode, were encountered at 0.5 to $1 \mathrm{~mm}$ along the trajectory between the area of decreased and disappeared neural activity in 9 patients (Cases 1, 4-9, 11, and 12). The electrophysiological findings along the trajectory in each patient are summarized in Fig. 4. The area of injury discharge was included in that of decreased neural activity in this study. We could clearly distinguish the location of the tumor based on these electrophysiological findings.

Tumor biopsy was performed in the region of disappeared neural activity including the surrounding components of the tumor. Because insertion of the biopsy needle into the cyst might have resulted in insufficient sampling of the tumor in Case 10, biopsy was performed within both the region of decreased neural activity and that of disappeared neural activity based on the MRI and electrophysiological findings. The T2-weighted and diffusionweighted MRI studies had shown high-intensity lesion surrounding the enhanced lesion in Case 11, so biopsy was performed within the region of the T2-weighted MRI high-intensity lesion (decreased and burst neural activity) as well as within the region of enhancing lesion (disappeared neural activity) because both samples were necessary for diagnosis.

\section{Correlations Between Electrophysiological, MRI, and Pathological Findings}

Preoperative MRI studies obtained with Gd demonstrated enhancement of the lesion in all 12 cases; homogeneous in 5 cases (Cases 3-5, 9, and 12) and heterogeneous in 7 cases (Cases 1, 2, 6-8, 10, and 11). The neural activity had disappeared in the tumor in all cases regardless of the enhancement pattern. Histological examination found that the samples taken within the region of disappeared neural activity consisted of tumor tissue in all 12 cases.

Decreased neural activity was observed from $6.3 \pm 4.5$ $\mathrm{mm}$ (range 2-18.4 $\mathrm{mm}$ ) above the point of disappearance of neural activity in all cases. Burst neural activity was observed from $3 \pm 1.4 \mathrm{~mm}$ (range 1.5-4.5 mm) above the point of decreased neural activity in 6 of 9 cases, in which the trajectory passed through the thalamus. The T2-weighted MRI studies showed a high-intensity area around the enhanced lesion in all of these 6 cases. Only 1 of the other 3 cases without burst neural activity showed such an area. The correlation between the appearance of burst neural activity and the presence of high-intensity area surrounding the enhanced lesion on T2-weighted MRI was studied using Fisher's exact test. The appearance of burst neural activity had a tendency to correlate with the presence of high-intensity area surrounding the enhanced lesion $(p=0.083)$. Tissue samples were obtained from regions of burst and decreased neural activity in Case 11 with malignant lymphoma. In this case, invasion of tumor cells at a much lower density was observed in the tissue sample taken from the region of burst and decreased neural activity (Fig. 5).

The histological diagnosis in the 6 cases with burst discharge was malignant lymphoma in 3 cases, and anaplastic astrocytoma, anaplastic oligodendroglioma, and germinoma in 1 case each. The histological diagnosis in the 3 cases without burst discharge was pilocytic astrocytoma in 2 cases and ganglioglioma in 1 case (Fig. 4).

\section{Illustrative Cases}

\section{Case 6 (Burst Type)}

The patient was a 24-year-old man whose MRI studies (Fig. 2B-F) showed the tumor with ringlike or multicentric enhancement surrounding a nonenhanced region in the left thalamus to midbrain. We planned selective removal of the ring-enhanced lesion and set the tentative target point in the nonenhanced center of the lesion. After passing through the internal capsule, the electrode had reached the thalamus at $17.5 \mathrm{~mm}$ from the tentative target point. Burst neural activity was observed frequently from $14.5 \mathrm{~mm}$ to $10 \mathrm{~mm}$. Neural activity began to decrease from $10 \mathrm{~mm}$ and finally disappeared from $4 \mathrm{~mm}$ to -2 $\mathrm{mm}$ (Fig. 2A). After the side hole of the biopsy needle 


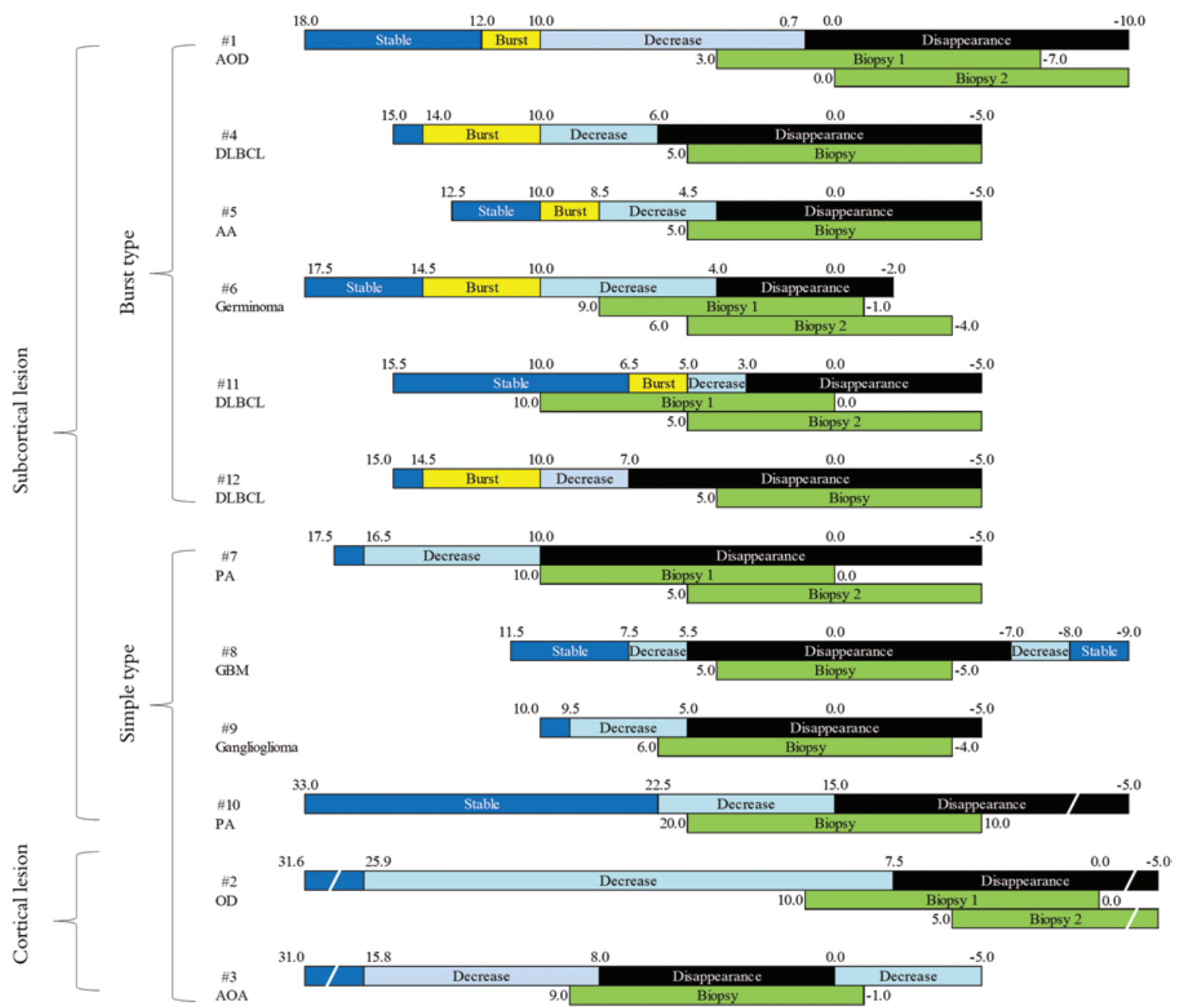

FIG. 4. Schematic representation of the relationship between the electrophysiological finding, the tissue sampling area, and the final pathological diagnosis. Numerical value on the scheme of neural activity or beside the sampling area shows distance from the tentative target point in millimeters. Blue label shows stable neural activity, yellow label shows burst discharge, light blue label shows decreased neural activity, and black label shows disappeared neural activity. Green label shows sampling area of the tumor. $\mathrm{AA}=$ anaplastic astrocytoma; $\mathrm{AOA}=$ anaplastic oligoastrocytoma; $\mathrm{AOD}=$ anaplastic oligodendroglioma; $\mathrm{GBM}=$ glioblastoma; $\mathrm{OD}=$ oligodendroglioma; $\mathrm{PA}=$ pilocytic astrocytoma .

was placed from $9 \mathrm{~mm}$ to $-1 \mathrm{~mm}$, samples mainly including the enhanced lesion were obtained by rotating the biopsy needle at this distance. Additional samples were obtained from $6 \mathrm{~mm}$ to $-4 \mathrm{~mm}$. Pathological examination of permanent sections confirmed the diagnosis as germinoma (Fig. 2G).

\section{Case 7 (Simple Type)}

The patient was a 17-year-old boy whose MRI studies (Fig. 3B-D) showed the tumor as a ring-enhanced region in the right thalamus to hypothalamus. After passing through the caudate nucleus and internal capsule, neural activity increased in the thalamus. Neural activity began to decrease at $16.5 \mathrm{~mm}$, and disappeared from $10 \mathrm{~mm}$ to $-5 \mathrm{~mm}$ (Fig. 3A). Biopsy samples were obtained from 10 $\mathrm{mm}$ to $0 \mathrm{~mm}$ and from $5 \mathrm{~mm}$ to $-5 \mathrm{~mm}$. Pathological examination of rapid-frozen sections provided a diagnosis of LGG and permanent sections confirmed pilocytic astrocytoma (Fig. 3E).

\section{Case 10 (Tissue Sample With Decreased and Disappeared Neural Activity)}

The patient was a 66-year-old man whose MRI studies (Fig. 6A and B) showed a multicentric tumor in the left temporal lobe and midbrain. Surgical removal of the left temporal lobe tumor was performed, and the histological diagnosis was anaplastic astrocytoma. Stereotactic biopsy was performed for histological diagnosis of the midbrain tumor. The tumor was a cystic lesion with only enhancement of the capsule. We planned selective biopsy of this enhanced lesion and set the tentative target at the center of the tumor. The microelectrode reached the thalamus at $33 \mathrm{~mm}$ from the tentative target. Neural activity began to decrease at $22.5 \mathrm{~mm}$ and disappeared from $15 \mathrm{~mm}$ to -5 $\mathrm{mm}$. The patient's MRI studies had shown that the cystic wall was approximately $10 \mathrm{~mm}$ from the center of the tumor and the enhancement of the capsule wall was 4-5 mm thick, the outer edge of which was estimated to be approximately $15 \mathrm{~mm}$ and the inner one $10 \mathrm{~mm}$ from the target 

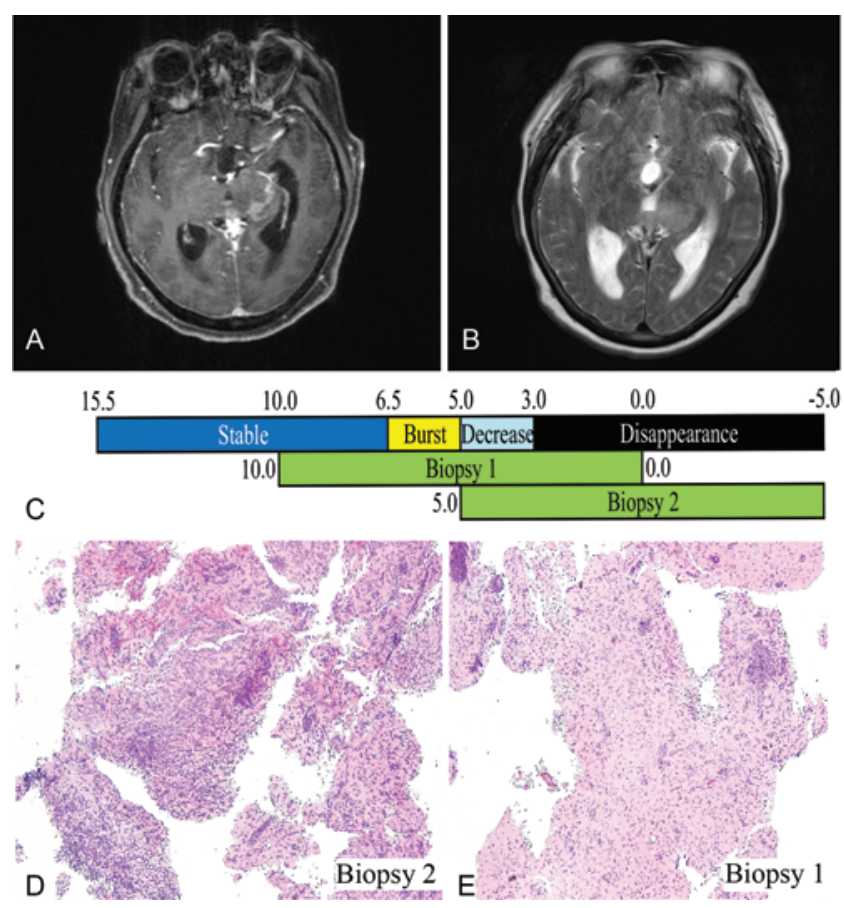

FIG. 5. Case 11. Electrophysiological, MRI, and pathological findings. A and B: Preoperative axial T1-weighted MR image with Gd (A) and T2-weighted MR image (B). C: Schematic representation of the relationship between electrophysiological findings and the tissue sampling area. D and E: Photomicrographs of samples obtained for histological examination of biopsy area 2 (D) demonstrating malignant lymphoma, and of biopsy area $1(\mathrm{E})$ demonstrating cell invasion with much lower density. $H \& E$, original magnification $\times 10(D$ and $E$ ). Figure is available in color online only.

point. Biopsy was performed at range from $20 \mathrm{~mm}$ to 10 $\mathrm{mm}$ to include both regions of decreased and disappeared neural activity (Fig. 6D) and to avoid obtaining only cystic content. Actually, solid component without cystic fluid was found in the biopsy samples. Pathological examination of permanent sections confirmed that the diagnosis was pilocytic astrocytoma, and the sample contained tumor and normal brain tissue with an obvious boundary (Fig. 6C).

\section{Case 9 (Smallest Tumor)}

The patient was a 7-year-old boy whose T1-weighted MRI studies obtained with Gd (Fig. 7A, C, and D) showed a left thalamic lesion with a maximum diameter of 10 $\mathrm{mm}$. Selective biopsy was planned to obtain a sample of the enhanced lesion with the tentative target in the center of the enhanced lesion. After passing through the internal capsule, the electrode had reached the thalamus at $10 \mathrm{~mm}$. Neural activity began to decrease from $9.5 \mathrm{~mm}$ and disappeared from $5 \mathrm{~mm}$ to $-5 \mathrm{~mm}$. Biopsy was performed from $6 \mathrm{~mm}$ to $-4 \mathrm{~mm}$ (Fig. 7E). Pathological examination of rapid-frozen and permanent sections provided a diagnosis of ganglioglioma (Fig. 7B). As mentioned above, burst neural activity was not found within the tumor.

\section{Discussion}

\section{Diagnostic Yield}

Previous series of stereotactic biopsy have included more than 150 patients since 2005, and have achieved diagnostic yields from $82.95 \%$ to $98.37 \%$. $^{6,8-11,14,20,27,39}$ The size and location of tumors were quite different in the 32 cases without microrecording compared with the 12 cases with microrecording. Despite the larger tumors in the former, histological diagnosis could not be confirmed in 2 cases, with a diagnostic yield per patient of $93.8 \%$. Our present technique with the aid of microrecording resulted in a diagnostic yield of $100 \%$, because we could distinguish tumor and normal tissue in real time during surgery by using data from both imaging and neural activity monitoring. The samples were obtained at the point identified as tumor based on the disappearance of neural activity. The diagnostic yield for most cases of HGG has been increased by selective sampling of marginal components because these tumors show high tumoral activity in the outer layer, whereas most of the inner component of tumor consists of necrosis. ${ }^{10}$ The present technique could selectively sample the marginal component by identifying the border of the tumor based on neural activity monitoring.

\section{Postoperative Complication and Mortality Rate}

In the present study a postoperative complication oc-

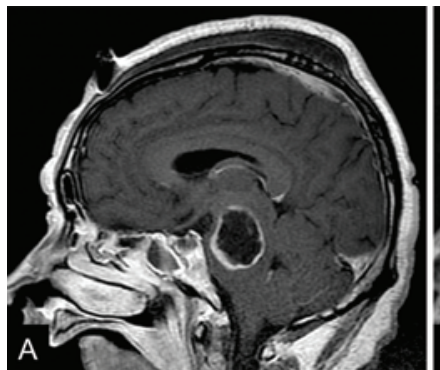

33.0

$\mathrm{D}$

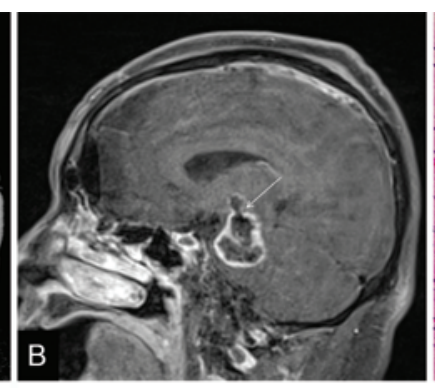

22.5
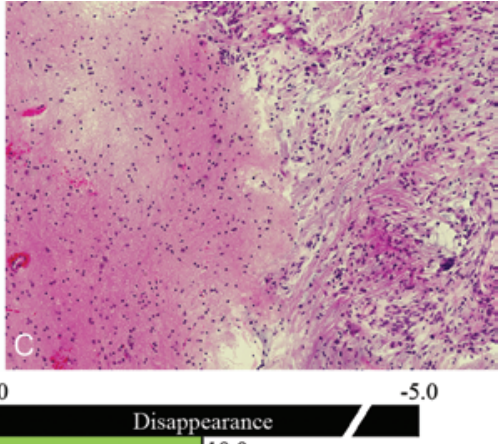

FIG. 6. Case 10. Electrophysiological, MRI, and pathological findings. A: Preoperative sagittal T1-weighted MR image with Gd. B: Postoperative T1-weighted MR image with Gd. Arrow shows sampling area of the tumor. C: Photomicrograph of a sample obtained for histological examination demonstrating both normal brain (left side of panel) and tumor tissue (right side of panel) with an obvious boundary. $H \& E$, original magnification $\times 20$. D: Schematic representation of the relationship between electrophysiological findings and the tissue sampling area. Figure is available in color online only. 

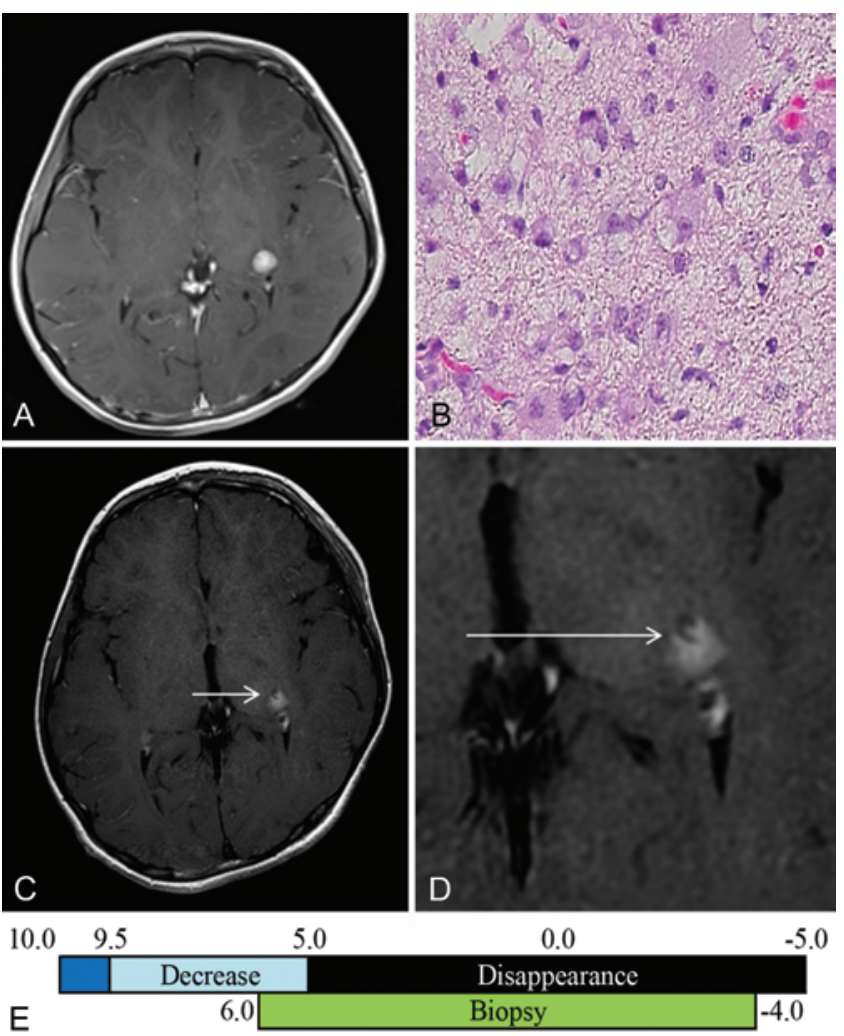

FIG. 7. Case 9. Electrophysiological, MRI, and pathological findings. A: Preoperative axial T1-weighted MR image with Gd. B: Photomicrograph of a sample obtained for histological examination demonstrating typical ganglioglioma. $\mathrm{H} \& \mathrm{E}$, original magnification $\times 40$. C and $\mathrm{D}$ : Postoperative axial T1-weighted MR image with $\mathrm{Gd}(\mathrm{C})$ and the magnified view (D). Arrows show sampling area of the tumor. E: Schematic representation of the relationship between electrophysiological findings and the tissue sampling area. Figure is available in color online only.

curred transiently, in only 1 patient, and the mortality rate was $0 \%$. Previous series of stereotactic biopsy including more than 150 patients since 2005 had postoperative complication rates from $2.9 \%$ to $12.1 \%$, and mortality rates from $0.6 \%$ to $1.34 \% .^{6,8-11,14,20,27,39}$ Mortality rates are higher in cases of thalamic or basal ganglia lesion, and complication rates are increased in cases of brainstem lesion..$^{8,14}$ In the present study, the mortality rate was $0 \%$ and the symptomatic hemorrhage rate was $0 \%$ despite the target tumors located in the thalamus, midbrain, or cortex. These rates are similar to the mortality rate of $0 \%$ and complication rate of $3.1 \%$ in our series of image-guided stereotactic biopsy without deep microrecording, which included 32 cases with tumor not located in deep brain structures. In the present study, postoperative transient sensory disturbance occurred only in Case 11. In this case, the biopsy sample was obtained in the region showing stable, burst, and decreased neural activity; therefore, the procedure clearly affected the function of normal brain.

\section{Associations Between Electrophysiological, MRI, and Pathological Findings}

In all cases neural activity had disappeared in the tumor, as shown in our previous preliminary study, ${ }^{29}$ re- gardless of the enhancement pattern, and diagnostic yield was $100 \%$ using the samples obtained from the region of disappeared neural activity. Moreover, neural activity disappeared in the tumor regardless of the histological type. The following 2 cases clearly demonstrated the association between electrophysiological, MRI, and pathological findings in the area where the neural activity disappeared and its surrounding area.

In a patient with pilocytic astrocytoma (Case 10), neural activity disappeared at $15 \mathrm{~mm}$ from the target point and a biopsy sample was obtained at a range from $20 \mathrm{~mm}$ to 10 $\mathrm{mm}$ by using the $10-\mathrm{mm}$ side-hole-length biopsy needle. In the surgical sample intended to obtain tissue consisting equally of the decreased neural activity area and the disappeared neural activity area, histological examination confirmed that the sample contained both normal brain and tumor tissue with an obvious boundary. The MRI studies showed that the enhanced lesion thickness around tumor cyst was approximately $5 \mathrm{~mm}$, which corresponded to the region with disappeared neural activity. These observations suggest that neural activity has disappeared in the ring-enhanced region, which was proved to be a tumor tissue in the case of LGG.

In a patient with malignant lymphoma (Case 11), samples of both regions of disappeared neural activity and decreased and burst neural activity were obtained separately. Histological examination confirmed that the density of tumor cells was obviously lower in the region of decreased and burst neural activity compared with the region of disappeared neural activity, indicating that decreased and burst neural activity corresponds to tumor cell invasion. These findings indicate that the regions of decreased neural activity have different pathological features in LGG and malignant lymphoma. A PET study performed using amino acid tracer has revealed tumor cell invasion in the high-intensity area on T2-weighted imaging around enhanced tumor on T1-weighted MRI studies obtained with Gd. ${ }^{40}$ Further investigation is needed to identify any correlation between the electrophysiological and MRI findings around the tumor.

\section{Algorithm to Identify Tumors With High Specificity}

We tried to develop an algorithm to identify tumors with high specificity based on the sequential electrophysiological findings. If the depth microrecording reveals that the neural activity begins to decrease along the trajectory during surgery, this indicates that the electrode is contacting the surrounding area of the tumor and is approaching the margin of tumor core. Sometimes, burst discharges are identified on the outer marginal zone of the area of decreased neural activity, which is strongly suggestive of malignancy in the tumor. After passing through the area of decreased neural activity, neural activity disappears. However, neural activity is often found only during the insertion of the electrode, which we call injury discharge, and occurs on the narrow border zone between the area of decreased and disappeared neuronal activity. This area may consist of a few neurons and a quite high density of tumor cells, so that electrical activity is found only during insertion of the electrode. After passing through this narrow border zone, neural activity disappears at the margin of the 


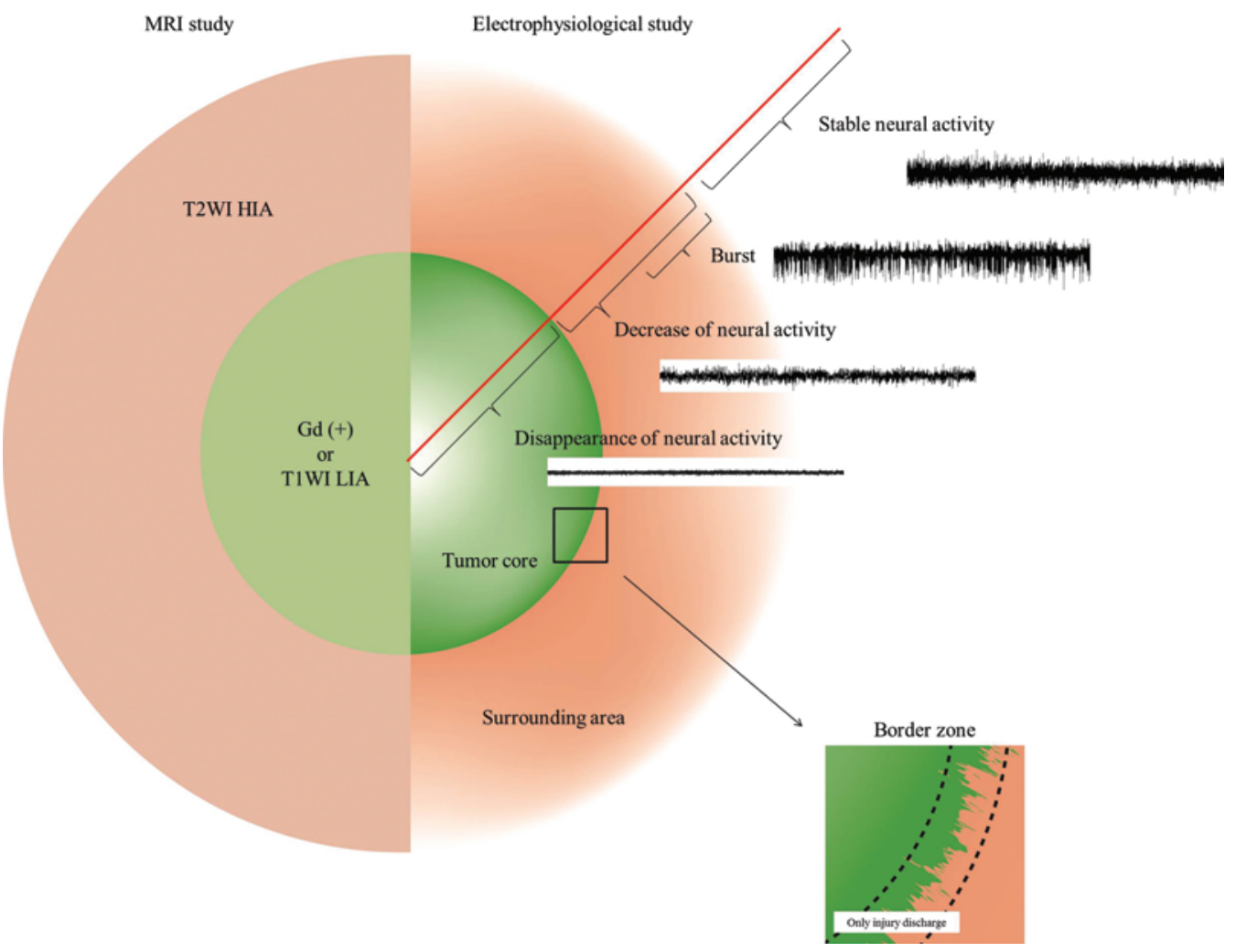

FIG. 8. Schematic representation of the proposed algorithm to identify tumors with high specificity. HIA = high-intensity area; LIA = low-intensity area; T1WI, T2WI = T1-, T2-weighted image.

tumor core. This effect is more obvious in cases of LGG. A schematic representation of our proposed algorithm to identify tumors with high specificity is shown in Fig. 8.

\section{Epileptogenicity of Brain Tumor}

Burst discharges were detected in the marginal zone of the area of decreased neural activity along the trajectory during surgery in 6 of 12 cases. Such electrophysiological findings and the MRI and histological data in our study might suggest methods for in vivo investigation of cases of epileptogenicity of brain tumor. The electrophysiological and histological study revealed that the neural activity decreased on the area of tumor invasion or peritumoral edema and also demonstrated that burst neural activity in the thalamus tended to correlate with the presence of a high-intensity area on T2-weighted MRI surrounding the enhanced lesion and usually encountered outside the decreased neural activity area, if present. Burst neural activity was considered to be encountered in the outer zone of high-intensity area on T2-weighted MRI. In the tissue surrounding the tumor, particularly malignant tumor, all neuronal function decreased, but imbalance of excitatory and inhibitory neuronal activity might occur in the outer zone. ${ }^{2}$

Experimental study indicated that decreased inhibition in the hippocampus caused disinhibition, whether of the inhibitory $\gamma$-aminobutyric acidergic neurons or the surrounding neurons that activate the inhibitory neuron remains unclear, and was involved in the pathophysiology of the epileptic state in the experimental epilepsy rat model. ${ }^{36}$ Also, selective degeneration of inhibitory $\gamma$-aminobutyric acidergic interneurons following hypoxia may be related to the development of epilepsy. ${ }^{35}$ Moreover, experimental study of the vulnerability of neuronal cells in the brain suggested that inhibitory neurons are easily damaged and degenerated in the thalamic reticular nucleus following transient ischemia due to raised intracranial pressure. ${ }^{33}$ Therefore, if the activity of inhibitory neurons is relatively more decreased than that of excitatory neurons, hyperexcitation of excitatory neurons might occur due to disinhibition in the outer zone of the high-intensity area on T2-weighted MRI, resulting in appearance of burst discharge. Such electrically unstable conditions might spread to the surrounding brain structures through fiber connections, causing the epileptogenic state in the brain. Recent clinical study of the seizure outcome after surgery for LGG demonstrated the importance of peritumoral condition, shown as a high-intensity area in T2-weighted MRI, for controlling postoperative epileptic seizure. ${ }^{16}$ Further investigation in vivo is needed to clarify this epileptogenic mechanism of the tumor.

\section{Indications and Limitations of Our Technique}

The error of MRI navigation at the target point is 4.1$5.9 \mathrm{~mm} .{ }^{4,34}$ Moreover, accurate positioning information is difficult to obtain in real time during surgery using only MRI-guided stereotactic biopsy. The present technique provided accurate positional information based on deep brain activity microrecording in real time during surgery and was useful for biopsy of thalamic or small brainstem 
tumor, in which sampling tissue is difficult to obtain..$^{8,14}$ Biopsy of the intended tissue could be performed safely and definitively, even in cases of small tumor with a diameter of $10 \mathrm{~mm}$ or narrow target tumor with only enhanced capsule. Selective removal of the most malignant components is necessary for accurate diagnosis of glioma, ${ }^{13,28,30,32}$ which was possible with our technique because we can distinguish the tumor boundary based on deep brain activity microrecording.

This study indicates that selective biopsy at the boundary of the region of disappeared neural activity increases the accuracy of biopsy targeting within glioma, although the electrophysiological characteristics might vary with the histological type or invasiveness of the tumor. These advantages can increase the diagnostic yield. The tumor was less easy to distinguish in biopsy samples of cortical lesion than in subcortical lesion, because the changing pattern of neural activity along the trajectory varied depending on the location of the tumor and the starting point of recording. Moreover, the tumor was less easy to distinguish in the deep cortical lesion because gray matter and white matter were arranged alternately along the trajectory, and neural activity was found in the former but not in the latter. We recommended surgery with local anesthesia in patients with good performance status for optimum neural activity recording, but we were able to obtain adequate neural activity recording for successful biopsy under general anesthesia in 4 patients.

\section{Conclusions}

Image-guided stereotactic biopsy performed using depth microrecording was safe, provided accurate positional information in real time, and could distinguish the brain tumor from normal brain structures during surgery. Moreover, this technique has a potential use for studying the epileptogenicity of the brain tumor.

\section{Acknowledgment}

We thank Dr. Akio Takahashi (Department of Neurosurgery, National Center Hospital, National Center of Neurology and Psychiatry, Kodaira, Tokyo, Japan) for the introduction of patients and technical assistance.

\section{References}

1. Allouch H, Pfeifenbring S, Behnke-Mursch J, Halatsch ME, Mursch K: Real-time ultrasound monitoring during intracranial needle biopsies: operative results and detection of complications in 100 cases. World Neurosurg 82:202-206, 2014

2. Beaumont A, Whittle IR: The pathogenesis of tumour associated epilepsy. Acta Neurochir (Wien) 142:1-15, 2000

3. Bekelis K, Radwan TA, Desai A, Roberts DW: Frameless robotically targeted stereotactic brain biopsy: feasibility, diagnostic yield, and safety. J Neurosurg 116:1002-1006, 2012

4. Breit S, LeBas JF, Koudsie A, Schulz J, Benazzouz A, Pollak P, et al: Pretargeting for the implantation of stimulation electrodes into the subthalamic nucleus: a comparative study of magnetic resonance imaging and ventriculography. Neurosurgery 58 (1 Suppl):ONS83-ONS95, 2006

5. Burkhardt JK, Neidert MC, Woernle CM, Bozinov O, Bernays RL: Intraoperative low-field MR-guided frameless stereotactic biopsy for intracerebral lesions. Acta Neurochir (Wien) 155:721-726, 2013
6. Chen CC, Hsu PW, Erich Wu TW, Lee ST, Chang CN, Wei $\mathrm{KC}$, et al: Stereotactic brain biopsy: single center retrospective analysis of complications. Clin Neurol Neurosurg 111:835-839, 2009

7. Czyż M, Tabakow P, Weiser A, Lechowicz-Głogowska BE, Zub LW, Jarmundowicz W: The safety and effectiveness of low field intraoperative MRI guidance in frameless stereotactic biopsies of brain tumours-design and interim analysis of a prospective randomized trial. Neurosurg Rev 37:127-137, 2014

8. Dammers R, Haitsma IK, Schouten JW, Kros JM, Avezaat CJJ, Vincent AJ: Safety and efficacy of frameless and framebased intracranial biopsy techniques. Acta Neurochir (Wien) 150:23-29, 2008

9. Dammers R, Schouten JW, Haitsma IK, Vincent AJ, Kros JM, Dirven CMF: Towards improving the safety and diagnostic yield of stereotactic biopsy in a single centre. Acta Neurochir (Wien) 152:1915-1921, 2010

10. Ersahin M, Karaaslan N, Gurbuz MS, Hakan T, Berkman MZ, Ekinci O, et al: The safety and diagnostic value of frame-based and CT-guided stereotactic brain biopsy technique. Turk Neurosurg 21:582-590, 2011

11. Ferreira MP, Ferreira NP, Pereira Filho AA, Franciscatto AC: Stereotactic computed tomography-guided brain biopsy: diagnostic yield based on a series of 170 patients. Surg Neurol 65 Suppl 1:S1:27-S1:32, 2006

12. Gralla J, Nimsky C, Buchfelder M, Fahlbusch R, Ganslandt O: Frameless stereotactic brain biopsy procedures using the Stealth Station: indications, accuracy and results. Zentralbl Neurochir 64:166-170, 2003

13. Greene GM, Hitchon PW, Schelper RL, Yuh W, Dyste GN: Diagnostic yield in CT-guided stereotactic biopsy of gliomas. J Neurosurg 71:494-497, 1989

14. Grossman R, Sadetzki S, Spiegelmann R, Ram Z: Haemorrhagic complications and the incidence of asymptomatic bleeding associated with stereotactic brain biopsies. Acta Neurochir (Wien) 147:627-631, 2005

15. Guiot G, Derome P, Arfel G, Walter S: Electrophysiological recording in stereotaxic thalamotomy for parkinsonism. Prog Neurol Surg 5:189-221, 1973

16. Ius T, Pauletto G, Isola M, Gregoraci G, Budai R, Lettieri $\mathrm{C}$, et al: Surgery for insular low-grade glioma: predictors of postoperative seizure outcome. J Neurosurg 120:12-23, 2014

17. Kandel EI, Schavinsky YV: Stereotaxic apparatus and operations in Russia in the 19th century. J Neurosurg 37:407-411, 1972

18. Kim JE, Kim DG, Paek SH, Jung HW: Stereotactic biopsy for intracranial lesions: reliability and its impact on the planning of treatment. Acta Neurochir (Wien) 145:547-555, 2003

19. Kondziolka D, Lunsford LD: The role of stereotactic biopsy in the management of gliomas. J Neurooncol 42:205-213, 1999

20. Kongkham PN, Knifed E, Tamber MS, Bernstein M: Complications in 622 cases of frame-based stereotactic biopsy, a decreasing procedure. Can J Neurol Sci 35:79-84, 2008

21. Krieger MD, Chandrasoma PT, Zee CS, Apuzzo ML: Role of stereotactic biopsy in the diagnosis and management of brain tumors. Semin Surg Oncol 14:13-25, 1998

22. Leksell L: A stereotaxic apparatus for intracerebral surgery. Acta Chir Scand 99:229-233, 1949

23. Leksell L, Leksell D, Schwebel J: Stereotaxis and nuclear magnetic resonance. J Neurol Neurosurg Psychiatry 48:1418,1985

24. Li CL, van Buren JM: Micro-electrode recordings in the brain of man with particular reference to epilepsy and dyskinesia, in Somjen GG (ed): Neurophysiology Studied in Man. Amsterdam: Excerpta Medica, 1972, pp 49-63 
25. Maroon JC, Bank WO, Drayer BP, Rosenbaum AE: Intracranial biopsy assisted by computerized tomography. J Neurosurg 46:740-744, 1977

26. Marossero F: The importance of neurophysiological tests for the localization of the targets in stereotaxic neurosurgery. Prog Neurol Surg 5:355-386, 1973

27. McGirt MJ, Woodworth GF, Coon AL, Frazier JM, Amundson E, Garonzik I, et al: Independent predictors of morbidity after image-guided stereotactic brain biopsy: a risk assessment of 270 cases. J Neurosurg 102:897-901, 2005

28. Mosskin M, Ericson K, Hindmarsh T, von Holst H, Collins VP, Bergström M, et al: Positron emission tomography compared with magnetic resonance imaging and computed tomography in supratentorial gliomas using multiple stereotactic biopsies as reference. Acta Radiol 30:225-232, 1989

29. Ohye C, Shibazaki T, Hirai T, Matsumura M, Kawashima Y, Hirato M: Microrecording for the study of thalamic organization, for tumor biopsy and removal. Stereotact Funct Neurosurg 52:136-144, 1989

30. Pauleit D, Floeth F, Hamacher K, Riemenschneider MJ, Reifenberger G, Müller HW, et al: O-(2-[18F]fluoroethyl)-Ltyrosine PET combined with MRI improves the diagnostic assessment of cerebral gliomas. Brain 128:678-687, 2005

31. Quinn J, Spiro D, Schulder M: Stereotactic brain biopsy with a low-field intraoperative magnetic resonance imager. Neurosurgery 68 (1 Suppl Operative):217-224, 2011

32. Revesz T, Scaravilli F, Coutinho L, Cockburn H, Sacares P, Thomas DG: Reliability of histological diagnosis including grading in gliomas biopsied by image-guided stereotactic technique. Brain 116:781-793, 1993

33. Ross DT, Duhaime AC: Degeneration of neurons in the thalamic reticular nucleus following transient ischemia due to raised intracranial pressure: excitotoxic degeneration mediated via non-NMDA receptors? Brain Res 501:129-143, 1989

34. Shamir RR, Joskowicz L, Spektor S, Shoshan Y: Target and trajectory clinical application accuracy in neuronavigation. Neurosurgery 68 (1 Suppl Operative):95-102, 2011

35. Sloper JJ, Johnson P, Powell TP: Selective degeneration of interneurons in the motor cortex of infant monkeys following controlled hypoxia: a possible cause of epilepsy. Brain Res 198:204-209, 1980

36. Sloviter RS: Decreased hippocampal inhibition and a selective loss of interneurons in experimental epilepsy. Science 235:73-76, 1987

37. Spiegel EA, Wycis HT, Marks M, Lee AJ: Stereotaxic apparatus for operations on the human brain. Science 106:349350,1947

38. Takizawa T, Soto S, Sanou A, Murakami Y: Frameless isocentric stereotactic laser beam guide for image-directed microsurgery. Acta Neurochir (Wien) 125:177-180, 1993

39. Teixeira MJ, Fonoff ET, Mandel M, Alves HL, Rosemberg S: Stereotactic biopsies of brain lesions. Arq Neuropsiquiatr 67:74-77, 2009

40. Whitfield GA, Kennedy SR, Djoukhadar IK, Jackson A: Imaging and target volume delineation in glioma. Clin Oncol (R Coll Radiol) 26:364-376, 2014

41. Zernov DN: [Encephalometer: device for estimation of parts of brain in human.] Proc Soc Physicomed Moscow Univ 2:70-80, 1889 (Russian)

\section{Author Contributions}

Conception and design: Hirato, Iijima, Yoshimoto. Acquisition of data: Hirato, Iijima, Miyagishima, Horiguchi, Sugawara, Hirato, Yokoo. Analysis and interpretation of data: Hirato, Iijima, Miyagishima, Hirato, Yokoo. Drafting the article: Hirato, Iijima, Yoshimoto. Critically revising the article: Hirato, Yoshimoto. Reviewed submitted version of manuscript: Hirato, Yoshimoto. Approved the final version of the manuscript on behalf of all authors: Hirato. Statistical analysis: Iijima. Administrative/techni$\mathrm{cal} /$ material support: Hirato, Iijima, Miyagishima, Hirato, Yokoo. Study supervision: Hirato, Yoshimoto.

\section{Correspondence}

Masafumi Hirato, Department of Neurosurgery, Gunma University Graduate School of Medicine, 3-39-22 Showa-machi, Maebashi, Gunma 371-8511, Japan. email: mfhirato@gunma-u.ac.jp. 